\title{
CEFALÉIA PÓS-TRAUMÁTICA CRÔNICA EM TRAUMATISMOS CRÂNIO-ENCEFÁLICOS LEVES
}

\author{
JANO ALVES DE SOUZA*, PEDRO FERREIRA MOREIRA FILHO**, CARLA DA CUNHA JEVOUX***
}

\begin{abstract}
RESUMO - Apesar de as evidências atuais indicarem que a cefaléia pós-traumática crônica (CPTc) resulta de alterações orgânicas, os sofredores dessa condição continuam sendo, ainda, frequentemente, considerados tão somente como neuróticos ou simuladores, especialmente, quando a dor se origina de TCE leves. O objetivo deste estudo foi identificar as características clínicas da CPTc após traumatismos crânio-encefálicos (TCE) leves. Foram estudados 27 pacientes de 16 a 64 anos que preencheram os critérios estabelecidos para CPTc e TCE leve. Verificou-se o início da cefaléia no mesmo dia do TCE em 51,8\% dos indivíduos. As seguintes formas clínicas foram identificadas: migrânea (70,3\%); cefaléia do tipo tensional - CTT $(51,8 \%)$ e cefaléia cervicogênica $(11,1 \%)$. A associação de CTT e migrânea ocorreu em 29,6\%. Trinta e três por cento dos trabalhadores, $40 \%$ das donas de casa e $50 \%$ dos estudantes da amostra manifestaram comprometimento da capacidade produtiva. Apesar disso, apenas três pacientes $(11,1 \%)$ encontravam-se em litígios por compensação. A ausência de potencial de ganho por parte da maioria dos pacientes e a uniformidade do quadro clínico apresentado são sugestivos de uma causa orgânica.
\end{abstract}

PALAVRAS-CHAVE: cefaléia, traumatismo craniano leve.

\section{Chronic post-traumatic headache after mild head injuries}

ABSTRACT - Current evidence indicates that chronic post-traumatic headache (cPTH) has organic causes. Nevertheless, these patients are considered as neurotics or malingering by health professionals, mainly if the headache originates from mild head injuries (MHI). Our aim was to identify the features of cPTH after MHI. We studied 27 consecutive patients fulfilling the criteria stablished for cPTH and MHI. Headache began on the same day of the trauma in $51.8 \%$ of patients. The clinical features allowed the following diagnosis: migraine (70.3\%); tension type headache $(51.8 \%)$; cervicogenic headache $(11.1 \%)$. Concomitance of migraine and tension type headache was found in $29.6 \%$. Thirty three percent of employees, $40 \%$ of housewives and $50 \%$ of students in our series referred prejudice in their productive activities. However, only three patients $(11.1 \%)$ were claiming for compensation. The lack of potential gain and the uniformity of the clinical presentation are suggestive that the cPTH has an organic cause.

KEY WORDS: headache, mild head injury.

A classificação de 1988 da Sociedade Internacional de Cefaléia (SIC) ${ }^{1}$ reservou o capítulo 5 para as cefaléias pós-traumáticas (CPT). De acordo com esta classificação, considera-se CPT aquela que se inicia até 14 dias após um traumatismo da cabeça, excluindo-se as cefaléias pré-existentes que se acentuam com o traumatismo e aquelas decorrentes dos hematomas intracranianos ou da hidrocefalia pós-traumática. As CPT podem ser subdivididas em agudas ou crônicas. Nesses casos, é necessário acrescentar-se um segundo dígito (5.1 e 5.2, respectivamente). Consideram-se agudas (CPTa) aquelas que desaparecem dentro de até oito semanas do traumatismo e crônicas (CPTc) as que persistem por período de tempo maior do que este. De acordo com a gravidade do traumatismo que as originou, as CPT podem ser classificadas como relacionadas a trauma craniano importante e/

Ambulatório de Cefaléias do Serviço de Neurologia da Universidade Federal Fluminense (UFF), Niterói RJ: *Mestre em Neurologia; **Professor Adjunto da Disciplina de Neurologia da UFF, Responsável pelo Ambulatório de Cefaléias do Serviço de Neurologia da UFF; ***Mestre em Neurologia. Aceite: 24-novembro-1998.

Dr. Jano Alves de Souza - Rua Tavares de Macedo 121/801 - 24220-210 Niterói RJ - Brasil. E-mail: jasouza@bigfoot.com 
ou com sinais comprobatórios (5.1.1 ou 5.2.1) ou relacionadas a trauma craniano leve e/ou sem sinais comprobatórios (5.1.2 ou 5.2.2). São consideradas evidências de trauma craniano importante e/ou com sinais comprobatórios a ocorrência de perda de consciência, a amnésia pós-traumática de mais de 10 minutos ou alterações em pelo menos dois dos seguintes ítens: exame clínico; exame neurológico; RX do crânio; exames de neuroimagem; potenciais evocados; exame do líquor; testes de função vestibular ou testes neuropsicológicos. As CPT podem ainda ser classificadas, de acordo com as características clínicas da dor, através da adição de um quarto dígito, da seguinte maneira: 1 - migrânea; 2 - cefaléia do tipo tensional; 3 - cefaléia em salvas; 4 - tipo aumento da pressão intracraniana; 5 - tipo diminuição da pressão intracraniana; 6 - tipo lesão local; 7 - tipo vasodilatação; 8 - tipo facada; 9 - outros tipos; 10 - dois ou mais tipos.

Os dados sobre a proporção de pacientes com traumatismos crânio-encefálicos (TCE) que desenvolvem cefaléia recorrente são discrepantes. Brenner et al. ${ }^{2}$, estudando vítimas de TCE de todos os níveis de gravidade, encontraram esta queixa em 32\% dos pacientes. Na amostra de Edna \& Cappelen ${ }^{3}$ com predomínio de TCE leves, $23 \%$ dos pacientes referiram cefaléia recorrente, três a cinco anos após o traumatismo. Rimel et al. ${ }^{4}$ e Levin et al. ${ }^{5}$, estudando apenas TCE leves encontraram, após três meses do traumatismo, índices de respectivamente $78 \%$ e $47 \%$. De acordo com o comentário contido na introdução ao capítulo 5 da classificação da SIC, uma relação causal entre o trauma craniano e a dor é frequente nos traumas cranianos importantes e/ou com sinais comprobatórios e infrequente nos traumatismos leves e/ou sem sinais comprobatórios ${ }^{1}$. Por outro lado, Yamaguchi ${ }^{6}$ observou relação inversa entre a gravidade do TCE e a incidência de CPTc.

CPT é uma designação genérica que engloba quadros com patogenia e manifestações clínicas diferentes ${ }^{7}$. Entre as formas clínicas da CPTc nem todas podem ser classificadas perfeitamente utilizando-se apenas a relação do quarto dígito da classificação da SIC $^{8-12}$. É o caso da cefaléia cervicogênica, frequentemente relacionada com traumatismos e cujos critérios diagnósticos foram estabelecidos em 1990 por Sjaastad et al. ${ }^{13}$. Além dessas, algumas formas especiais de CPTc têm sido descritas. A cefaléia no local da lesão ("site of injury headache ") foi descrita inicialmente por Simons \& Wolf ${ }^{14}$ e confirmada por Vijayan \& Watson ${ }^{15}$. Caracteriza-se por dor de caráter variável, localizada sobre uma área restrita do couro cabeludo ou face correspondente ao local de traumatismo da cabeça e onde se pode, com frequência, encontrar uma cicatriz. Ao exame, a área pode revelar anestesia ou hiperestesia, além de alterações da função sudomotora. A dor responde satisfatoriamente à administração de carbamazepina ou fenitoína, confirmando o seu caráter neuralgiforme. As cefaléias disautonômicas pós-traumáticas são formas menos frequentes de CPTc. Constituem grupo heterogêneo de cefaléias recorrentes que têm em comum o fato de iniciarem-se após traumatismo da cabeça e associarem-se a alterações autonômicas envolvendo as funções pupilar, lacrimal e sudomotora da face. São conhecidas três formas distintas, descritas respectivamente por Vijayan \& Dreyfus ${ }^{16}$, Khurana \& Nirankari ${ }^{17}$ e Khurana ${ }^{18}$.

A CPTc pode manifestar-se como sintoma isolado ou como parte da síndrome pós-traumática (SPT), um conjunto de sintomas somáticos, cognitivos, emocionais e comportamentais que pode se desenvolver após TCE dos mais variados níveis de gravidade, inclusive leves s,10,19. $^{9}$.

A causa da SPT e da CPT têm sido objeto de muita discussão entre os que as consideram manifestações de neurose ou de simulação e aqueles que propõem uma causa orgânica ${ }^{20,21}$. Até a primeira metade deste século, as teorias psicogênicas preponderaram. No entanto, com o desenvolvimento da neuropatologia, neuropatologia experimental e neuropsicologia, multiplicaramse os achados sugestivos da organicidade dessas desordens ${ }^{22-25}$. Apesar disso, ainda é grande o desconhecimento acerca desses temas em nosso meio e os pacientes continuam sendo, com frequência, considerados pelos profissionais da saúde, tão somente como neuróticos ou simuladores em busca de compensações financeiras. A descrença quanto à veracidade das queixas desses pacientes é tanto maior quanto menor a gravidade do TCE que as originou. Nosso objetivo foi estudar clinicamente indivíduos com CPTc relacionada a TCE leves. 


\section{PACIENTES E MÉTODO}

Foram estudados 27 pacientes consecutivos atendidos no Ambulatório de Cefaléias do Hospital Universitário Antônio Pedro (Universidade Federal Fluminense), sendo 14 mulheres e 13 homens, com idade variando de 16 a 64 anos (média 32 anos) e que preencheram os critérios estabelecidos para TCE leve e CPTc.

Os critérios utilizados foram:

Para TCE leve: 1) período de inconsciência ou amnésia pós-traumática menor do que 30 minutos; 2) resultado inicial na Escala de Coma de Glasgow entre 13 e 15 sem deterioração posterior; 3) exame neurológico normal; 4) ausência de fraturas com afundamento no exame radiológico simples de crânio; 5) ausência de lesões intracranianas na tomografia axial computadorizada de crânio para os pacientes que apresentaram perda da consciência de qualquer duração.

Para CPTc: 1) início da cefaléia até 14 dias após o TCE; 2) persistência da cefaléia por mais de oito semanas; 3) Ausência de cefaléia recorrente anterior ao TCE.

Critérios adicionais: 1) não estar sob efeito de álcool ou drogas psicoativas por ocasião do TCE; 2) não ter apresentado, juntamente com o TCE, outras lesões orgânicas capazes de contribuir para a piora do nível de consciência por choque ou alterações metabólicas; 3) ter, pelo menos, dois episódios de cefaléia por mês, desde o TCE até a primeira consulta.

Através de entrevista pessoal, foram obtidas informações acerca dos seguintes itens: 1) dados pessoais; 2) características do TCE; 3) características da cefaléia; 4) outros sintomas da SPT associados; 5) comprometimento das atividades profissional, escolar ou doméstica pela cefaléia ou outros sintomas pós-traumáticos persistentes; 6) envolvimento com litígios por compensação.

Para a caracterização do tipo de CPT, utilizamos, sempre que possível, a codificação do quarto dígito para as cefaléias dos grupos 5 a 11 da classificação da $\operatorname{SIC}^{1}$. Para os casos de cefaléia cervicogênica, foram utilizados os critérios de Sjaastad et al. ${ }^{13}$.

\section{RESULTADOS}

Quanto ao mecanismo básico do TCE, em 22 pacientes (88,8\%) este foi do tipo em que a cabeça com seus movimentos livres é submetida a aceleração, chocando-se contra um anteparo. Noutros quatro pacientes (14.8\%), o mecanismo foi o de golpe de um objeto em movimento contra a cabeça estacionária. Apenas uma paciente $(3.7 \%)$ sofreu trauma do tipo chicote cervical.

As causas de TCE apontadas foram: quedas $(n=11 ; 40,7 \%)$; acidentes de trânsito $(n=5$; $18,5 \%)$; atropelamentos $(n=4 ; 14,8 \%)$; queda de objetos sobre a cabeça $(n=3 ; 11,1 \%)$; coice de cavalo $(n=1 ; 3,7 \%)$, cabeçada em jogo de futebol $(n=1 ; 3,7 \%)$, mergulho em água rasa $(n=1$; $3,7 \%)$ e agressão $(n=1 ; 3,7 \%)$.

Onze pacientes $(40,7 \%)$ apresentaram perda da consciência imediatamente em seguida ao trauma; outros onze $(40,7 \%)$ mantiveram-se vigis, porém, manifestaram algum período de obnubilação ou amnésia pós-traumática. Os cinco restantes, não apresentaram perda da consciência, obnubilação ou amnésia pós-traumática.

A cefaléia iniciou-se no mesmo dia do TCE em 14 pacientes $(51,8 \%)$; entre o segundo e o sétimo dias em quatro (14,8\%); e entre o oitavo e o décimo-quarto dias em nove pacientes $(33,3 \%)$.

Estudando-se as características da cefaléia (localização, frequência, intensidade, duração das crises, tipo da dor, fatores precipitantes ou agravantes, fenômenos concomitantes e história familiar), encontramos os seguintes diagnósticos: migrânea sem aura; migrânea com aura, cefaléia do tipo tensional episódica; cefaléia do tipo tensional crônica e cefaléia cervicogênica (Fig. 1). A concomitância de mais de um tipo de cefaléia foi observada em nove pacientes (33,3\%). A associação de migrânea com ou sem aura com cefaléia do tipo tensional crônica foi a mais frequente, sendo encontrada em oito pacientes (29.6\%). Uma paciente (3.7\%) apresentou associação de cefaléia cervicogênica com migrânea. 


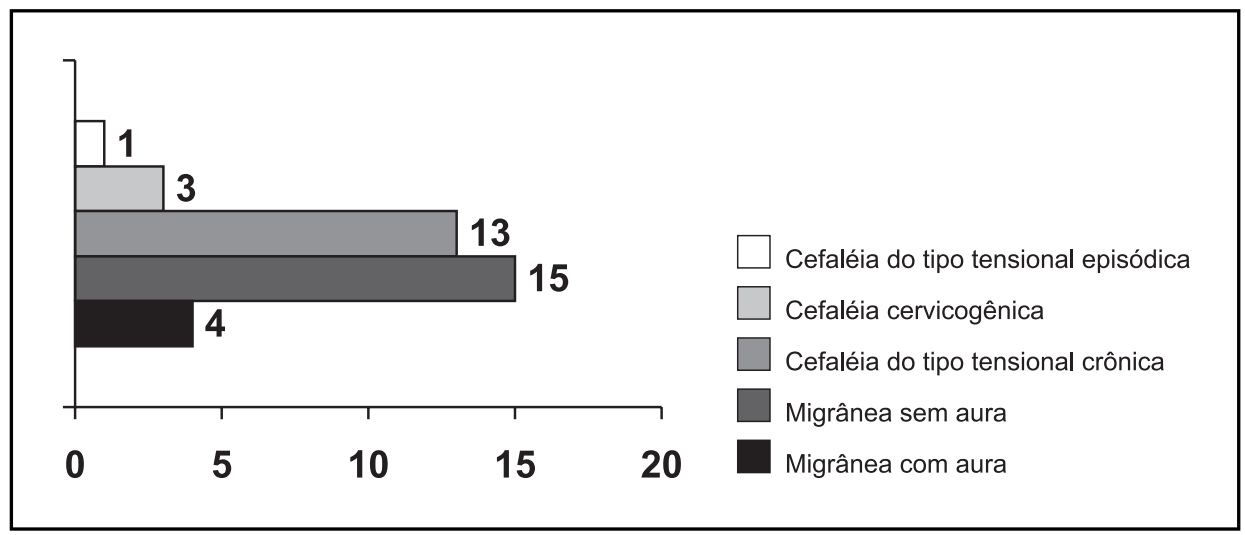

Fig 1. Tipos de Cefaléia.

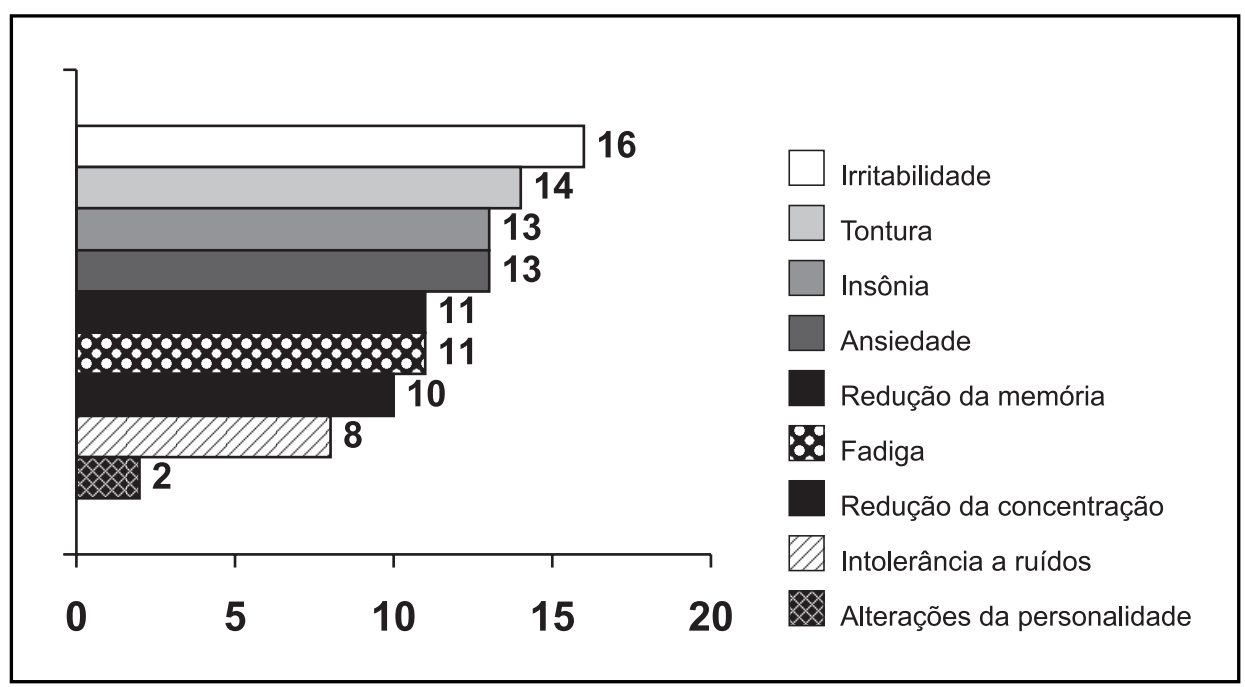

Fig 2. Sintomas pós-traumáticos.

Vinte e quatro $(88,8 \%)$ dos pacientes estudados relataram outros sintomas da síndrome póstraumática (Fig. 2).

Dentre os pacientes estudados, 18 encontravam-se empregados por ocasião do TCE. Destes, seis apresentaram redução da capacidade laborativa, necessitando afastar-se, por algum tempo, das atividades profissionais, sendo que três encontravam-se, ainda, afastados do trabalho quando da primeira entrevista. Interrogados sobre os sintomas que determinaram o afastamento do trabalho, cinco alegaram como o principal fator a cefaléia.

Havendo quatro estudantes na amostra, dois referiram redução no desempenho escolar e a necessidade de faltarem, com frequência, às aulas. Ambos informaram como principal causa a cefaléia.

Entre as donas de casa, em número de cinco na amostra, duas queixaram-se de redução da capacidade de gerenciamento do lar. Ambas referiram-se à cefaléia como a principal causa.

Três pacientes $(11,1 \%)$ buscaram compensação financeira através de litígios judiciais. Em nenhum dos casos, a sentença havia sido estabelecida por ocasião da entrevista. 


\section{DISCUSSÃO}

Um problema metodológico de muitos estudos sobre TCE leves é o emprego de critérios diagnósticos imprecisos, como por exemplo a utilização da Escala de Coma de Glasgow como único instrumento para a classificação da gravidade do $\mathrm{TCE}^{26}$. O mesmo se dá com os estudos acerca da CPTc, que incluem pacientes cuja dor precede ao traumatismo ou em que não se pode demonstrar correlação temporal nítida com o $\mathrm{TCE}^{27}$. Em nosso estudo, para a caracterização da gravidade dos TCE, utilizamos critérios rigorosos e em acordo com a literatura especializada recente ${ }^{26}$. Para o diagnóstico das CPT utilizamos os critérios da Sociedade Internacional de Cefaléias'.

Tanto a CPTc, quanto os demais sintomas da SPT parecem predominar no sexo feminino ${ }^{4,28}$. Barnat $^{29}$, estudando 117 pacientes com CPTc, encontrou discreto predomínio do sexo feminino $(54 \%)$. Já na amostra de migrânea pós-traumática de Weiss et al. ${ }^{8}$, o sexo feminino correspondeu a 77\% dos pacientes. Em nosso estudo encontramos distribuição semelhante entre os sexos. Talvez, isto se deva à maior incidência de TCE entre homens, conforme demonstrado por diversos estudos epidemiológicos ${ }^{29-32}$.

Nossos achados confirmam o que tem sido relatado na literatura, de que não é necessário haver a perda da consciência para que se desenvolva a $\mathrm{CPT}^{10}$.

Um dado marcante de nossa amostra é o início precoce da cefaléia, com cerca de metade dos pacientes apresentando cefaléia já no primeiro dia do TCE. Isto reforça a necessidade de se estabelecer como critério diagnóstico da CPT o início da dor em íntima relação temporal com o TCE ${ }^{1,27}$.

Quanto às formas de CPTc, nossos achados são concordantes com os da literatura, que se referem à migrânea e à cefaléia do tipo tensional como as formas mais frequentemente observadas ${ }^{9,10}$. Cabe ressaltar a grande frequência com que estas duas formas de cefaléia se associaram em nossos pacientes. Curiosamente, encontramos poucos casos de cefaléia cervicogênica, apesar da reconhecida correlação deste tipo de dor com antecedentes traumáticos ${ }^{25}$. Isto, talvez, decorra do fato de termos tido apenas uma paciente com história de chicote cervical, mecanismo mais frequentemente relacionado com esta forma de cefaléia. Não encontramos qualquer caso de cefaléia no local da lesão. Como esta forma de cefaléia pode ter início mais tardio, relacionado com o encarceramento de fibras sensitivas em tecido cicatricial e regeneração aberrante, a adoção dos critérios da SIC, que estabelecem o prazo de 14 dias como o máximo para o início da cefaléia, com certeza fizeram com que esses casos não fossem incluídos.

De acordo com os nossos achados, com frequência, a cefaléia e os demais sintomas da SPT foram suficientemente graves, ao ponto de provocarem redução da capacidade laborativa ou de aprendizado dos pacientes. A principal causa do comprometimento nestes pacientes foi, indiscutivelmente, a cefaléia.

Acreditando que os TCE leves não poderiam cursar com alterações estruturais, Miller $^{21}$ considerou que o envolvimento com litígios e o potencial de ganho financeiro seria fator preponderante para a perpetuação dos sintomas pós-traumáticos, inclusive a cefaléia. No entanto, estudos posteriores como o de Packard ${ }^{33}$ não confirmaram essa opinião. Outros estudos têm demonstrado a ocorrência de alterações estruturais microscópicas nos TCE leves ${ }^{23,24}$, o que permite especulações acerca de uma causa orgânica para a CPT e a síndrome pós-traumática. Em nossa casuística, apenas três pacientes encontravam-se envolvidos com litígios ou possuíam qualquer potencial de ganho, sugerindo, pelo menos, que este seja um fator pouco relevante para a perpetuação da CPT em nosso meio.

Avaliando os pacientes retrospectivamente, não verificamos características neuróticas prétraumáticas. Em todos eles, as dificuldades de adaptação ao trabalho, às atividades escolares ou domésticas, somente tiveram início após o traumatismo.

Na opinião dos autores, a uniformidade do quadro manifestado e a ausência de potencial de ganho para a maioria dos pacientes reforçam a hipótese de uma causa orgânica para a CPTc. 


\section{REFERÊNCIAS}

1. Headache Classification Committee of the International Headache Society (HCCIHS). Classification and diagnostic criteria for headache disorders, cranial neuralgias and facial pain. Cephalalgia 1988;8(Suppl 7):44-45.

2. Brenner C, Friedman AP, Merritt HH, Denny-Bown DE. Post-traumatic headache. J Neurosurg 1944;1:379-391.

3. Edna TH, Cappelen J. Late postconcussional symptoms in traumatic head injury: an analysis of frequency and risk factors. Acta Neurochir 1987;86:2-17.

4. Rimel RW, Giordani B, Barth JT, Boll TJ, Jane JA. Disability caused by minor head injury. Neurosurgery 1981;9:221-228.

5. Levin HS, Mattis S, Ruff RM, et al. Neurobehavioral outcome following minor head injury: a three-center study. J Neurosurg 1987;66:234-243.

6. Yamaguchi M. Incidence of headache and severity of head injury. Headache 1992;32:427-431.

7. Wessely P, Holzner F. On the problem of post-traumatic headache. Wien Klin Wochenschr 1987;99:549-554.

8. Weiss HD, Strn BJ, Goldberg J. Post-traumatic migraine: chronic migraine precipitated by minor head or neck trauma. Headache 1991;31:451-456.

9. Goldstein J. Posttraumatic headache and the postconcussion syndrome. Med Clin North Am 1991;75:641-651.

10. Evans RW. The postconcussion syndrome and the sequelae of mild head injury. Neurol Clin 1992;10:815-847.

11. Turkewitz LJ, Wirth O, Dawson GA, Casaly JS. Cluster headache following head injury: a case report and review of the literature. Headache 1992;32:504-506.

12. Reik L. Cluster headache after head injury. Headache 1987;27:509-510.

13. Sjaastad O, Fredriksen TA, Pfaffenrath V. Cervicogenic headache: diagnostic criteria. Headache 1990;30:725-726.

14. Simons DJ, Wolff HG. Studies on headache: mechanisms of chronic post-traumatic headache. Psychosom Med 1946;8:227-242.

15. Vijayan N, Watson C. Site of injury headache. Headache 1989;29:502-506.

16. Vijayan N, Dreyfus PM. Posttraumatic dysautonomic cephalalgia: clinical observations and treatment. Arch Neurol 1975;32:649-652.

17. Khurana RK, Nirankari VS. Bilateral sympathetic dysfunction in post-traumatic headaches. Headache 1986;26:183-188.

18. Khurana RK. Posttraumatic headache with ptosis, miosis and chronic forehead hyperhidrosis. Headache 1990;30:64-68.

19. Benton AL. Historical notes on the postconcussion syndrome. In Levin HS, Eisenberg HM, Benton AL (eds). Mild head injury. New York, Oxford University Press, 1989. Cap 1, 3-7.

20. Strauss I, Savitsky N. Head injury: neurologic and psychiatric aspects. Arch Neurol Psychiatry 1934;31:893-955.

21. Miller H. Accident neurosis. Br Med J 1961;1:992-998.

22. Strich SJ. Diffuse degeneration of the cerebral white matter in severe dementia following head injury. J Neurol Neurosurg Psychiatry 1956;19:163-185.

23. Oppenheimer DR. Microscopic lesions in the brain following head injury. J Neurol Neurosurg Psychiatry 1968;31:299-306.

24. Povlishock JT, Becker DP, Cheng CLY, Vaughan GW. Axonal change in minor head injury. J Neuropathol Exp Neurol 1983;42:225-242.

25. Speed WG III. Closed head injury sequelae: changing concepts. Headache 1989;29:643-647

26. Williams DH, Levin HS, Eisenberg HM. Mild head injury classification. Neurosurgery 1990;27:422-428.

27. Edmeads J. Headache and head injury... Post Hoc, or Propter Hoc? Headache 1988;28:228-229.

28. Rutherford WH. Postconcussion symptoms: relationship to acute neurological indices, individual differences, and circumstances of injury. In Leevin HS, Eisenberg HM, Benton A.L (eds.). Mild head injury. New York: Oxford Univ Press, 1989:217-228.

29. Barnat MR. Post-traumatic headache patients: I. Demographics, injuries, headache and health status. Headache 1986;26:271-277.

30. Kraus JF, Nourjah P. The epidemiology of mild head injury. In Levin HS, Eisenberg HM, Benton AL Mild Head Injury. New York: Oxford Univ Press, 1989:8-22.

31. Annegers JF, Grabow JD, Kurland LT, Laws ER. The incidence, causes, and secular trends of head trauma in Olmstead County, Minnesota, 1935-1974. Neurology 1980;30:912-919.

32. Whitman S, Coonley-Hoganson R, Desai BT. Comparative head trauma experiences in two socioeconomicaly different Chicago-Area communities: a population study. Am J Epidemiol 1990;119:570-580.

33. Packard RC. Posttraumatic headache: permanency and relationship to legal settlement.Headache 1992;32:496-500. 